Check for updates

Cite this: Nanoscale Adv., 2019, 1, 1482

\title{
DNA-templated Au nanoclusters coupled with proximity-dependent hybridization and guanine- rich DNA induced quenching: a sensitive fluorescent biosensing platform for DNA detection $\uparrow$
}

\begin{abstract}
Hai-Bo Wang, (DD * Hong-Yu Bai, Gao-Li Dong and Yan-Ming Liu
In this paper, the fluorescence signal of poly(A) DNA-templated Au nanoclusters (AuNCs) is found to be greatly quenched by photoinduced electron transfer (PET) when they are close to guanine (G)-rich DNA. Based on the findings, we have designed a low-cost fluorescence biosensing strategy for the sensitive detection of DNA. Highly luminescent and photo-stable poly(A) DNA-AuNCs were utilized as the fluorescent indicator and G-rich DNA was utilized as the fluorescent quencher. In the absence of target DNA, DNA-AuNCs failed to hybridize with the G-rich DNA and did not form the duplex DNA structure. Strong fluorescence intensity at $475 \mathrm{~nm}$ was observed due to the DNA-AuNCs being far away from the G-rich DNA. However, in the presence of target DNA, the DNA-AuNCs together with G-rich DNA could hybridize with the target DNA, leading to the $5^{\prime}$ terminus of the DNA-AuNCs and the $3^{\prime}$ terminus of Grich DNA being in close proximity and promoting the cooperative hybridization. Therefore, a " $\mathrm{Y}^{\text {" junction }}$ structure was formed and the G-rich sequences were brought close to the AuNCs. Therefore, the fluorescence intensity of the sensing system decreased significantly. Taking advantage of the poly $(A)$ DNA-templated Au nanoclusters and G-rich DNA proximity-induced quenching, the strategy could be extended to determine other biomolecules by designing appropriate sequences of DNA probes.
\end{abstract}

Received 10th October 2018

Accepted 28th January 2019

DOI: $10.1039 / c 8 n a 00278 a$

rsc.li/nanoscale-advances fundamental studies and the applications of DNA-templated metal nanoclusters.

For example, single-stranded cytosine (C)-rich DNA has been utilized as the template for the formation of $\mathrm{Ag}$ nanoclusters (AgNCs) ${ }^{6-8}$ The prepared AgNCs possessed a high quantum yield and photo-stability. However, it usually suffered from the need for a relatively expensive precursor $\left(\mathrm{AgNO}_{3}\right)$ and timeconsuming preparation procedure (over $24 \mathrm{~h}$ ). ${ }^{6}$ Recently, Mokhir and co-workers found that double-stranded DNA could serve as the template and support the formation of copper nanoclusters (CuNCs) in 10 min. ${ }^{9}$ Wang's group reported that single-stranded polythymine (poly T) DNA could also be used as a template for the formation of CuNCs. ${ }^{10}$ Although the preparation of poly T-templated CuNCs was very quick (only $5 \mathrm{~min}$ ), the fluorescence quantum yield of these CuNCs was rather low. In addition, the fluorescence signal of DNA-templated CuNCs could be stable for just $20 \mathrm{~min} .{ }^{11,12}$ Therefore, the photo-stability of CuNCs still needed to improve. Very recently, Tseng and coworkers found that polyadenine DNA was the effective template for the formation of fluorescent AuNCs by a UV-light-assisted reduction method. ${ }^{13}$ However, it needed incubation for $24 \mathrm{~h}$ under $305 \mathrm{~nm}$ UV light irradiation. Thus, the exploitation of simple synthesis of highly photo-stable fluorescent nanoclusters is still at an early stage. 
As a novel DNA-assisted analytical strategy, the proximitydependent hybridization assay has attracted increasing interest in the sensitive detection of DNA and protein. ${ }^{14-19}$ The proximity-dependent hybridization assay depended upon the simultaneous recognition of a target molecule (e.g. DNA or protein) by a pair of affinity probes. This resulted in the hybridization of terminus sequences of the affinity probe pair being in close proximity. On the basis of this principle, Jiang's group has designed some single-step and sensitive electrochemical methods for DNA and platelet-derived growth factor (PDGF) detection by using proximity-dependent surface hybridization assay. ${ }^{\mathbf{1 4 1 5 , 2 0}}$ Zhao and co-workers have developed a fluorescent biosensor for the multiplexed detection of protein biomarkers based on the fluorescence proximity immunoassay. ${ }^{17}$ The proximity-dependent hybridization assay was simple, rapid, sensitive, and selective, and exhibited a low background.

It has been reported that most fluorescent dyes can be strongly quenched by guanine (G) bases through photoinduced electron transfer (PET) between guanine bases and fluorophores. ${ }^{21,22}$ In the PET process, quenchable fluorophores act as electron acceptors in their excited state and guanine bases work as an electron donor. ${ }^{21}$ The fluorescence quenching phenomenon is usually called guanine $(G)$ quenching. Due to its cost-effective and easy design, G quenching of fluorescencelabeled DNA probes has been used as a powerful tool for the detection of metal ions, ${ }^{23-25}$ DNA, ${ }^{26,27}$ protein, ${ }^{28}$ adenosine triphosphate, ${ }^{29,30}$ exonuclease/endonuclease activity, ${ }^{31,32}$ and so on. However, most of these methods usually require complicated fluorescent dye labelling. Therefore, it is still highly desirable for the exploration of a low-cost and label-free Gquenching-based fluorescence strategy for bioassay.

Very recently, our group reported that poly(A) DNA-templated AuNCs exhibited a strong fluorescence signal and high photo-stability through heating-assisted reduction methods. ${ }^{33,34}$ In this work, we have demonstrated a new fact that G-rich DNA proximity could induce the fluorescence quenching of the DNAAuNCs. This phenomenon might be attributed to the photoinduced electron transfer (PET) process between the DNA-AuNCs and G-rich DNA in proximity, leading to a decrease in fluorescence intensity. Hence, a novel fluorescent biosensing platform has been developed for DNA detection by coupling the advantages of the poly(A) DNA-templated Au nanoclusters and G-rich DNA proximity-induced quenching.

\section{Experimental}

\subsection{Reagents}

$\mathrm{HAuCl}_{4}$ was purchased from Sigma Aldrich Chemical Co. (St. Louis, MO, USA). Trisodium citrate and other reagents (analytical reagent grade) were obtained from Sinopharm Chemical Reagent Co., Ltd. (Shanghai, China) and used without further purification. All buffers and reagent solutions were prepared with ultrapure water (with electrical resistance $>18.2 \mathrm{M} \Omega \mathrm{cm}$ ) from a Millipore Milli-Q water purification system (Billerica, MA, USA). All oligonucleotides used in this work were synthesized by Sangon Biotech Co., Ltd. (Shanghai, China). The sequences are shown in Table 1.

\subsection{Apparatus}

All fluorescence measurements were performed on a Hitachi F7000 fluorescence spectrometer (Hitachi, Japan). The fluorescence emission spectra were recorded from $400 \mathrm{~nm}$ to $560 \mathrm{~nm}$ with an excitation wavelength of $290 \mathrm{~nm}$. The excitation and emission slits were both set at $5 \mathrm{~nm}$. UV-vis absorption spectra were collected on a Hitachi U-3900 spectrophotometer (Hitachi, Japan). Transmission electron microscopy (TEM) images were obtained using a Tecnai G2 F20 S-TWIN field emission transmission electron microscope.

\subsection{Preparation of fluorescent DNA-AuNCs}

DNA-AuNCs were synthesized by using poly(A)-DNA as the template according to our previous reported methods. ${ }^{33,34}$ In brief, a $1 \mathrm{~mL}$ volume of an aqueous solution containing $50 \mu \mathrm{L} 1$ $\mathrm{mM} \mathrm{HAuCl}, 100 \mu \mathrm{L} 2 \mu \mathrm{M}$ P1 or P2 DNA probe and $100 \mu \mathrm{L} 50$ $\mathrm{mM}$ sodium citrate $(\mathrm{pH}$ 6) was prepared at room temperature. After that, the solution was incubated at $90{ }^{\circ} \mathrm{C}$ for $30 \mathrm{~min}$. Finally, the prepared AuNCs were named P1 or P2 DNA-AuNCs.

\subsection{Hybridization of DNA-AuNCs and G-rich DNA probe}

A series of guanine-rich DNA probes (3G, 4G, 5G, and 6G probes) was designed to hybridize with DNA-AuNCs and to quench the fluorescence of the as-prepared AuNCs. Briefly, $50 \mu \mathrm{L}$ P1 DNAAuNCs and $10 \mu \mathrm{L} 1 \mu \mathrm{M}$ G-rich DNA probe were added into a 100 $\mu \mathrm{L}$ reaction system (containing $20 \mathrm{mM}$ Tris- $\mathrm{HCl}$ and $100 \mathrm{mM}$ $\mathrm{NaCl}$; pH 7.4). The mixture was heated to $90^{\circ} \mathrm{C}$ for $1 \mathrm{~min}$ and then gradually cooled down to $37^{\circ} \mathrm{C}$. After being incubated at $37^{\circ} \mathrm{C}$ for $30 \mathrm{~min}$, the fluorescence emission spectra were immediately

Table 1 Sequences of oligonucleotides used in this work

\begin{tabular}{ll}
\hline DNA probes & Sequences $\left(5^{\prime} \rightarrow 3^{\prime}\right)$ \\
\hline P1 DNA & $5^{\prime}$-AAAAAAAAAAAAAAAAAAAAGTCAGTGTGGAAAATCTCTAGC-3' \\
3G probe & $5^{\prime}$-GCTAGAGATTTCCACACTGACT GGG-3' \\
4G probe & $5^{\prime}$-GCTAGAGATTTCCACACTGACT GGGG-3' \\
5G probe & $5^{\prime}$-GCTAGAGATTTCCACACTGACT GGGGG-3' \\
6G probe & $5^{\prime}$-GCTAGAGATTTCCACACTGACT GGGGGG-3' \\
P2 DNA & $5^{\prime}$-AAAAAAAAAAAAAAAAATATTCAAGCCGGAAATAGCAATAAGAC-3' \\
6G probe 2 & $5^{\prime}$-GGG TCA TCA AGA TAC AGC AAG AAG ATA GGGGGG-3' \\
Target DNA & $5^{\prime}$-G TCT TAT TGC TAT TTC CGG CTT GAA CTT CTT GCT GTA TCT TGA TGA CCC-3'
\end{tabular}


recorded on a Hitachi F-7000 fluorescence spectrometer with an excitation wavelength of $290 \mathrm{~nm}$.

\subsection{Fluorescent DNA assays}

In a typical DNA assay, $50 \mu \mathrm{L}$ P2 DNA-AuNCs, $10 \mu \mathrm{L} 1 \mu \mathrm{M} 6 \mathrm{G}$ probe 2 and $10 \mu \mathrm{L} \mathrm{H1N1}$ target DNA (different concentrations) were added into a $100 \mu \mathrm{L}$ reaction system (containing $20 \mathrm{mM}$ Tris- $\mathrm{HCl}$ and $100 \mathrm{mM} \mathrm{NaCl}$; $\mathrm{pH}$ 7.4). The mixture was heated to $90{ }^{\circ} \mathrm{C}$ for $1 \mathrm{~min}$ and then gradually cooled down to $37^{\circ} \mathrm{C}$. After being incubated at $37^{\circ} \mathrm{C}$ for $30 \mathrm{~min}$, the fluorescence intensity was immediately recorded on a Hitachi F-7000 fluorescence spectrometer with an excitation wavelength of $290 \mathrm{~nm}$.

\section{Results and discussion}

\subsection{Principle of the guanine proximity-induced fluorescence quenching}

As shown in Scheme 1A, P1 DNA was designed to have two parts. One part was the poly(A) DNA template as the AuNC sequences, the other was the hybridization sequences. The $6 \mathrm{G}$ probe also contained two parts, G-rich overhang sequences and hybridization sequences. The fluorescence signal of P1 DNA-AuNCs was quenched due to their proximity with G-rich sequences through DNA hybridization with the 6G probe. Taking advantage of the guanine proximity-induced fluorescence quenching and proximity-dependent hybridization assay, we designed a simple and single-step biosensing strategy for nucleic acid detection. The design principle is shown in Scheme 1B. In this sensing system, two oligonucleotide probes were designed according to the target DNA sequence and the melting temperatures. Firstly, P2 DNA, which was complementary to part of the target DNA, contained AuNC sequences and served as a signal probe. Secondly, $6 \mathrm{G}$ probe 2 , partially complementary with the target DNA and P2 DNA, acted as the quenching probe. As a proof-of-concept, an oligonucleotide sequence from influenza A virus (H1N1) was selected as the target DNA. The melting temperature for the hybrid of the P2 DNA and 6G probe 2 was calculated to be $-33.6{ }^{\circ} \mathrm{C}$ by using the Zuker program. ${ }^{35}$ Thus, in the absence of H1N1, P2 DNA failed to hybridize with the $6 \mathrm{G}$ probe 2 and did not form duplex DNA at a reaction temperature of $37{ }^{\circ} \mathrm{C}$. Strong fluorescence intensity at $475 \mathrm{~nm}$ was observed, because the P2 DNA-AuNCs were far away from the G-rich sequences. However, in the presence of target H1N1, the $6 \mathrm{G}$ probe 2 together with P2 DNA could hybridize with the target $\mathrm{H} 1 \mathrm{~N} 1$, respectively. Here, the $5^{\prime}$ terminus of the P2 DNA and the $3^{\prime}$ terminus of the $6 \mathrm{G}$ probe 2 have been drawn into close proximity, which promotes the cooperative hybridization of the residual complementary sequences of the $6 \mathrm{G}$ probe 2 and P2 DNA. Therefore, a "Y" junction structure formed and the Grich sequences were brought close to the P2 DNA-AuNCs, leading to strong fluorescence quenching. A low background was obtained by using the proximity-dependent hybridization.

\subsection{Characterization of poly(A) DNA-AuNCs}

The fluorescent DNA-AuNCs were successfully prepared by using poly(A) DNA as the synthesis template. ${ }^{33,34}$ The morphological characterization of the obtained AuNCs was performed by transmission electron microscopy (TEM). As shown in Fig. 1(A), the shapes of the prepared AuNCs were nearly spherical with an average diameter of $3 \mathrm{~nm}$. The fluorescence emission spectra of the prepared AuNCs were studied with different excitation wavelengths. From Fig. 1(B), it was observed that the emission peaks of AuNCs remained at around $475 \mathrm{~nm}$ as the excitation wavelength increased from $290 \mathrm{~nm}$ to $370 \mathrm{~nm}$. The highest fluorescence at $475 \mathrm{~nm}$ was obtained when the excitation wavelength was set at $290 \mathrm{~nm}$. The fluorescence quantum yield of the prepared AuNCs was 1.9\%. Additionally, the fluorescence intensity (at $475 \mathrm{~nm}$ ) of the prepared AuNCs had no obvious changes as the $\mathrm{pH}$ value was increased from 6.0 to 8.5 (shown in Fig. 1(C)). As indicated in Fig. 1(D), the

(A)

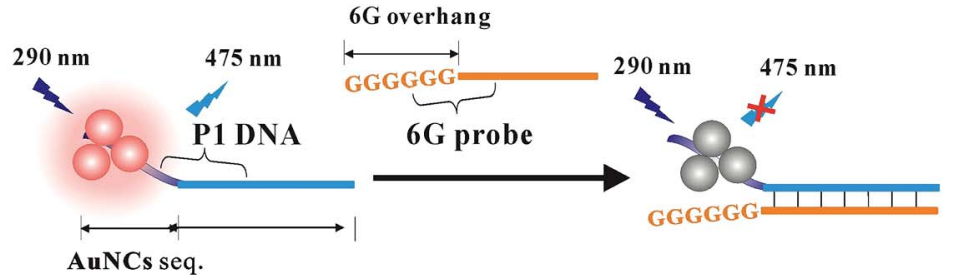

Hybridization seq.

(B)

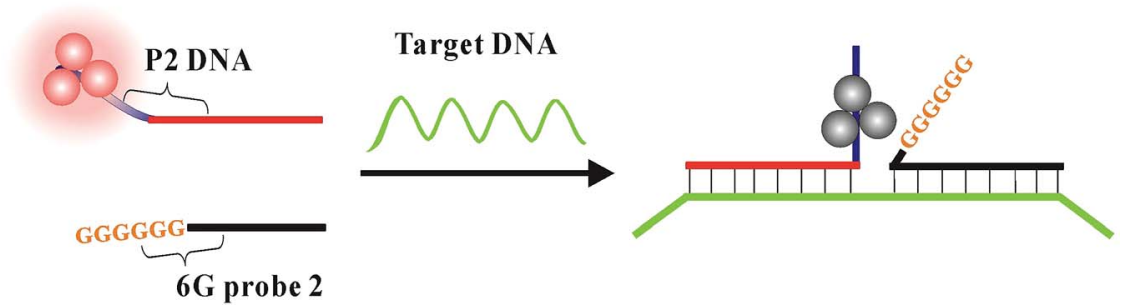

Scheme 1 (A) Schematic illustration of fluorescence quenching of P1 DNA-AuNCs through proximity with G-rich DNA. (B) Schematic illustration of a fluorescence biosensing method for DNA detection based on P2 DNA-AuNCs coupled with proximity-dependent hybridization and G-rich DNA induced quenching. 

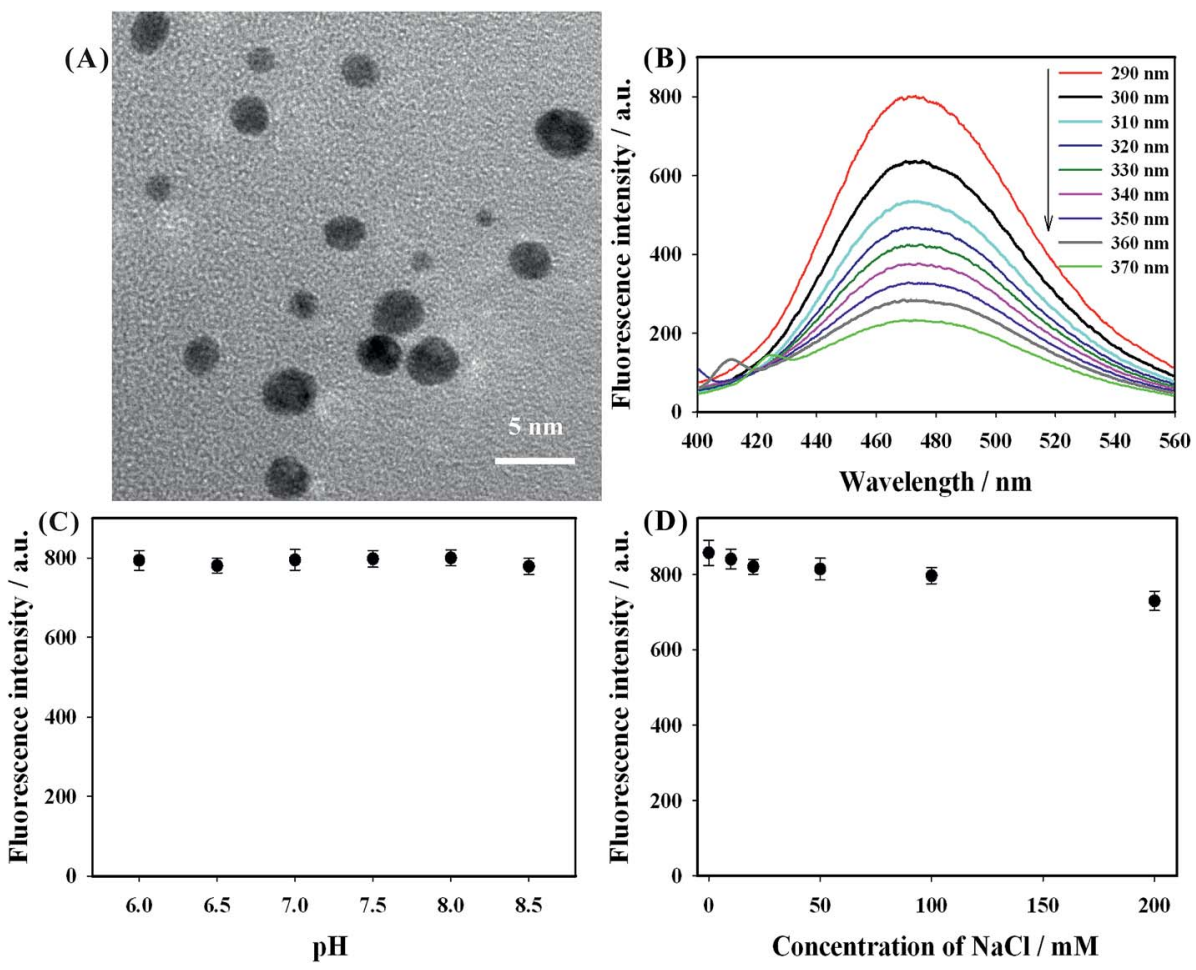

Fig. 1 (A) TEM image of the prepared DNA-AuNCs. (B) Fluorescence emission spectra of the DNA-AuNCs with different excitation wavelengths. (C) The effect of solution $\mathrm{pH}$ value on the fluorescence intensity of P1 DNA-AuNCs. (D) The effect of the NaCl concentration on the fluorescence intensity of P1 DNA-AuNCs.

fluorescence intensity of AuNCs showed no obvious decrease in the presence of $200 \mathrm{mM} \mathrm{NaCl}$. Furthermore, the fluorescence signal of the obtained AuNCs was very stable after being placed at $4{ }^{\circ} \mathrm{C}$ for 2 months. These results indicated that the DNAAuNCs possessed good stability and could be exploited as a fluorescent indicator for the development of a biosensing strategy.

\subsection{Evaluation of the feasibility of the fluorescence strategy}

In order to prove this quenching concept and evaluate the guanine proximity-induced fluorescence quenching, P1 DNAAuNCs were hybridized with different G-rich DNA probes (various guanine contents). The corresponding fluorescence emission spectra are shown in Fig. 2(A). Fig. 2(B) presents the fluorescence intensity (at $475 \mathrm{~nm}$ ) of P1 DNA-AuNCs hybridized with different G-rich DNA probes. It was observed that fluorescence intensity decreased obviously along with the increase of the number of guanine bases in proximity to the P1 DNAAuNCs. Approximately $70 \%$ of the fluorescence intensity was quenched when P1 DNA-AuNCs were hybridized with the 6G probe (containing 6 guanine bases). This may be ascribed to the close proximity between guanine bases and AuNCs, resulting in the photoinduced electron transfer and the decrease of fluorescence intensity. To further verify that the fluorescence quenching was the result of the guanine proximity caused by the hybridization process, the typical fluorescence intensity of AuNCs was recorded by a thermal cycling process. From Fig. 3, it was observed that fluorescence quenching came from the DNA hybridization, and the guanine proximity-induced fluorescence quenching was reversible.

To better understand the guanine proximity-induced fluorescence quenching, we investigated the fluorescence intensity of P1 DNA-AuNCs in the presence of different concentrations of 6G probe. Fig. 4(A) depicts the typical fluorescence emission spectra of P1 DNA-AuNCs with different concentrations of 6G probe. From Fig. 4(A), it could be clearly seen that the fluorescence signal decreased remarkably with the increase of the $6 \mathrm{G}$ probe concentration from 0 to $150 \mathrm{nM}$ (curve (a) to curve (j)). These results suggested that the more the $6 \mathrm{G}$ probe was present, the more duplex DNA formed, resulting in more guanine proximity and the decrease of fluorescence intensity. Fig. 4(B) shows the relationship between the fluorescence signal and the 6G probe concentrations. The efficient fluorescence quenching might be attributed to the photoelectron transfer (PET) between the guanine bases and the P1 DNA-AuNCs, which was similar to the mechanism of fluorophores being quenched by guanines. ${ }^{21,22}$ The mechanism of the fluorescence quenching was also investigated by performing some additional experiments. Firstly, the UV-vis absorption spectra of the $6 \mathrm{G}$ probe and the fluorescence emission spectra of P1 DNA-AuNCs were recorded. From Fig. S1 (ESI), $\dagger$ it was observed that there was no overlap between the absorption spectra of the $6 \mathrm{G}$ probe and the emission spectra of P1 DNA-AuNCs, which weakened the fluorescence resonance energy transfer (FRET) as a possible mechanism for the fluorescence quenching of AuNCs. Secondly, it was found that the absorption spectra of P1 DNA-AuNCs 

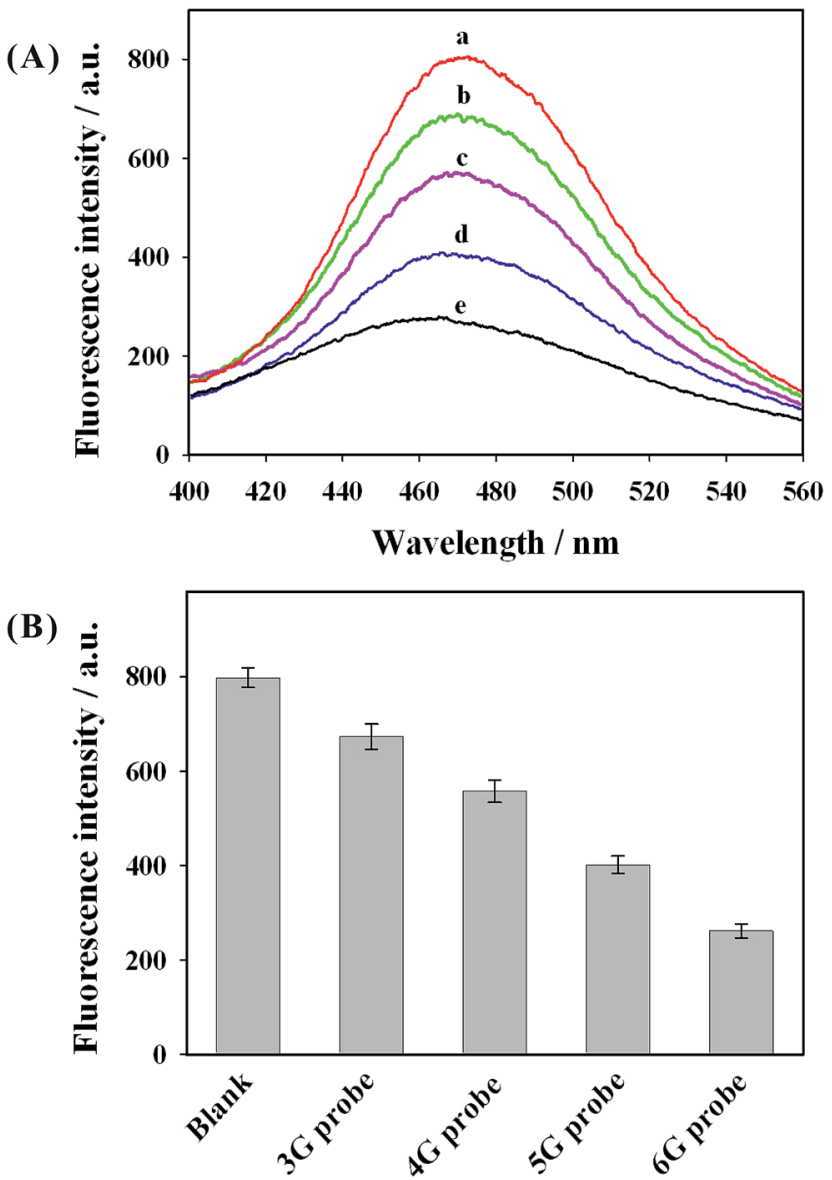

Fig. 2 (A) Fluorescence emission spectra of the P1 DNA-AuNCs hybridized with different G-rich DNA probes. The curves from (a) to (e) are those of the blank, 3G probe, 4G probe, 5G probe, and 6G probe, respectively. (B) The relationship between the fluorescence intensity and G-rich DNA probes.

showed no obvious change in the presence of $6 \mathrm{G}$ probe, thereby eliminating the possibility of surface reactions through noncovalent interactions as a possible mechanism. Thirdly, the zeta

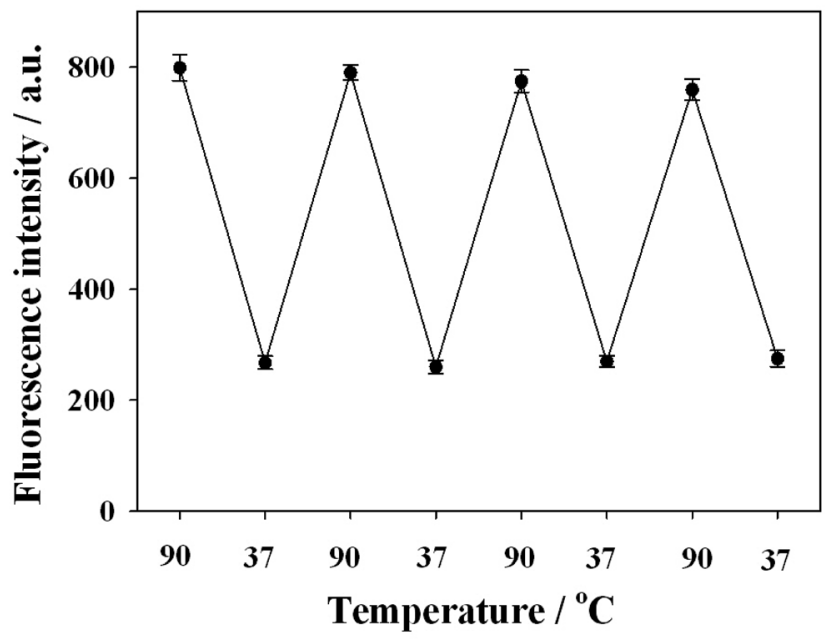

Fig. 3 Representative fluorescence trace of P1 DNA-AuNCs with the $6 \mathrm{G}$ probe during a thermal cycling process.
(A)

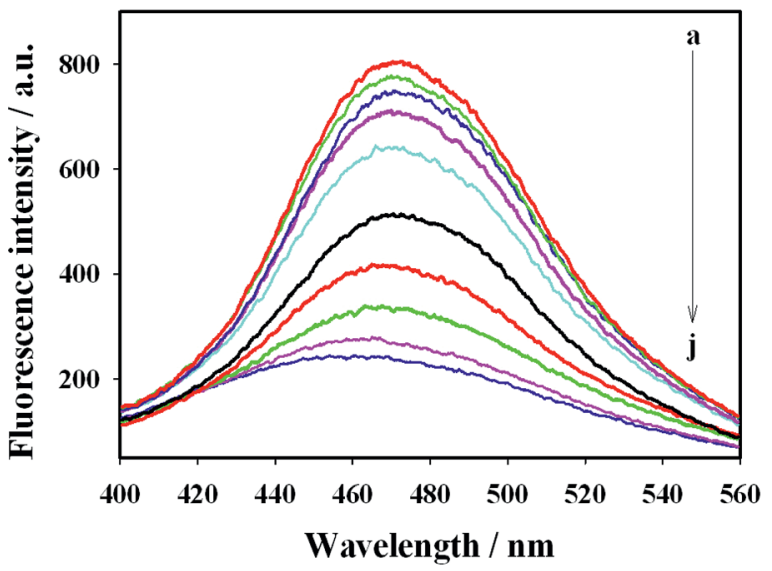

(B)

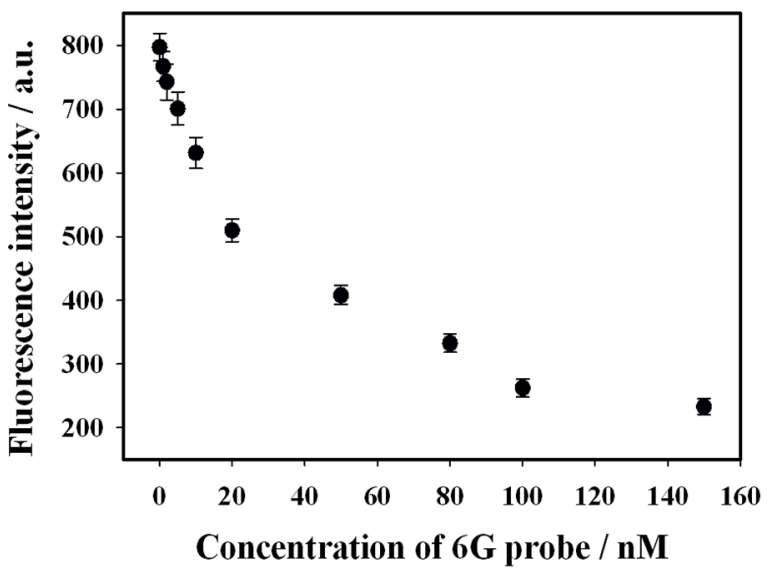

Fig. 4 (A) Fluorescence spectra of P1 DNA-AuNCs in the presence of increasing $6 \mathrm{G}$ probe concentrations: from curves (a) to (j), the concentration is $0,1,2,5,10,20,50,80,100$, and $150 \mathrm{nM}$, respectively. (B) The relationship between the fluorescence intensity and the concentration of $6 \mathrm{G}$ probe.

potential of P1 DNA-AuNCs in the absence and presence of $6 \mathrm{G}$ probe was also investigated. A significant reduction of the zeta potential of P1 DNA-AuNCs was obtained from $-23.6 \mathrm{mV}$ to $-14.5 \mathrm{mV}$ in the presence of $6 \mathrm{G}$ probe, indicating the strong electrostatic interaction between the P1 DNA-AuNCs and 6G probe. Therefore, the fluorescence quenching presumably resulted from the photoelectron transfer (PET) between the AuNCs and $6 \mathrm{G}$ probe, which was similar to some previously reported methods. ${ }^{22-24,32}$

\subsection{Analytical performance of DNA assay}

As shown in Fig. S2 (ESI), $\dagger$ the prepared P2 DNA-AuNCs exhibited strong fluorescence intensity at $475 \mathrm{~nm}$ (curve (a)). When 6G probe 2 was introduced, the fluorescence signal of P2 DNA-AuNCs showed no obvious change (curve (b) in Fig. S2 $\dagger$ ). When $100 \mathrm{nM}$ of target DNA H1N1 was present in the sensing system, the fluorescence intensity at $475 \mathrm{~nm}$ decreased obviously (curve (c) in Fig. S2†) because the target DNA H1N1 could hybridize with the $6 \mathrm{G}$ probe 2 and P2 DNA-AuNCs, respectively. Hence, a "Y" junction structure formed and the G-rich sequences were close to the AuNCs, leading to the fluorescence intensity being quenched. Fig. 5(A) displays the fluorescence emission spectra of the label- 
(A)

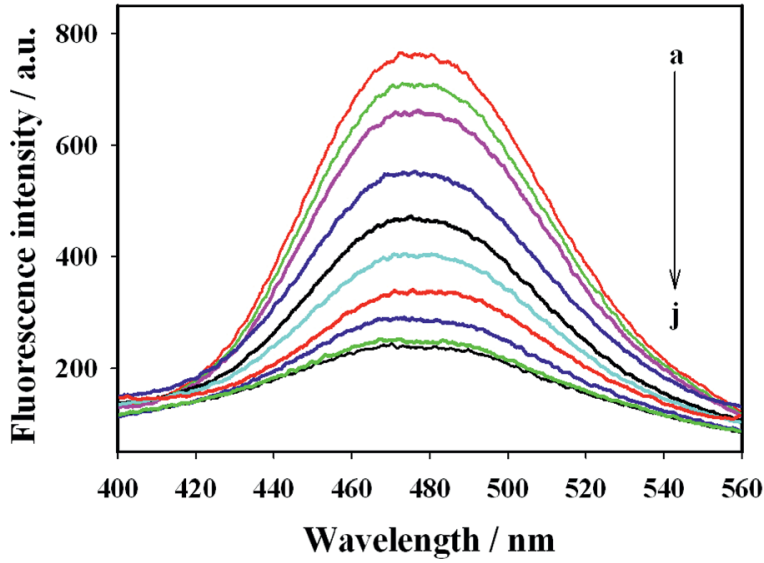

(B)

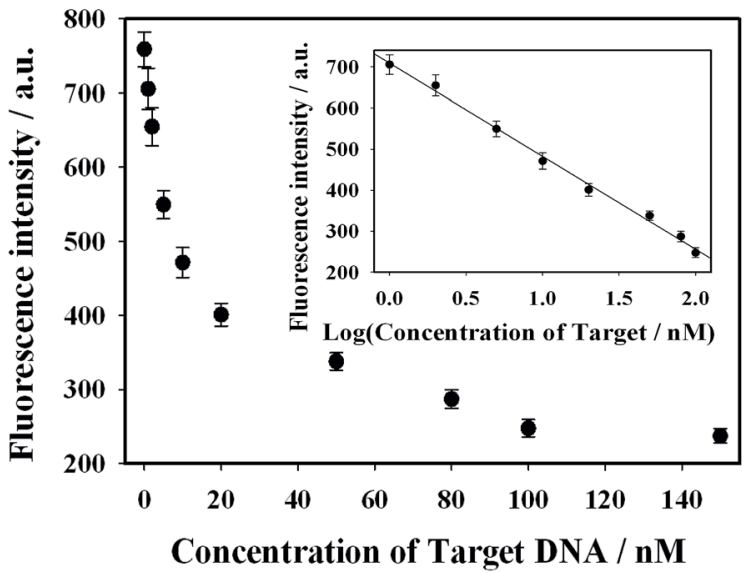

Fig. 5 (A) Fluorescence spectra of P2 DNA-AuNCs and 6G probe 2 in the presence of increasing target DNA concentrations: from curves (a) to (j), the concentration is $0,1,2,5,10,20,50,80,100$, and $150 \mathrm{nM}$, respectively. (B) The relationship between the fluorescence intensity and the target DNA concentration. The inset in (B) shows the calibration curve.

free fluorescence strategy in the presence of different concentrations of H1N1. It was clearly observed that the fluorescence intensity decreased obviously along with the increase of the H1N1 concentration from 1 to $150 \mathrm{nM}$ (from curve (a) to curve (j) in Fig. 5(A)). This result implied that when more target H1N1 was present, more G-rich sequences could be close to P2 DNA-AuNCs, leading to a decrease in fluorescence intensity. The inset in Fig. 5(B) shows a good linear relationship between fluorescence intensity $(475 \mathrm{~nm})$ and the logarithm of the H1N1 concentrations (from 1 to $100 \mathrm{nM})$. According to the rule $(\mathrm{S} / \mathrm{N}=3)$, the limit of detection (LOD) could be calculated to be $200 \mathrm{pM}$. The LOD was

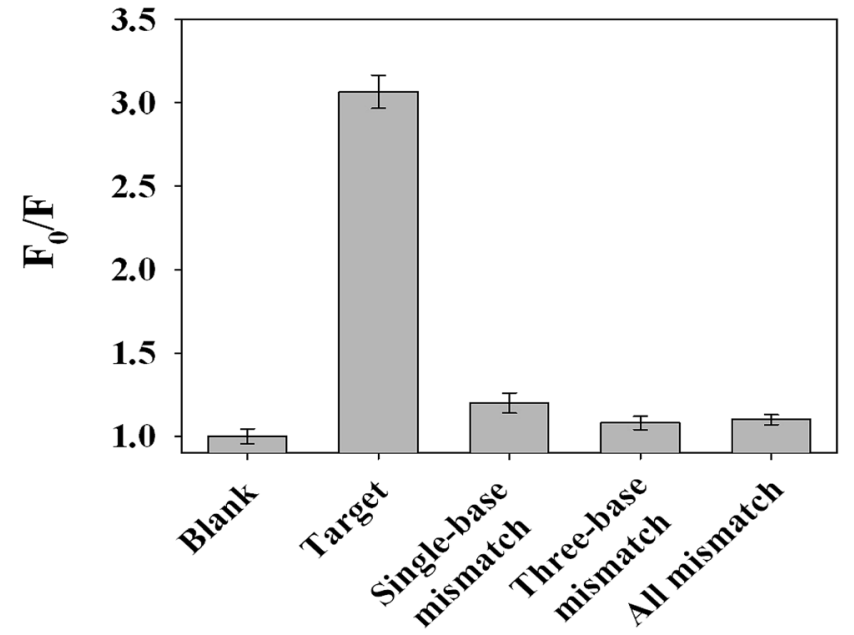

Fig. 6 Bar chart of the fluorescence responses of the blank, target DNA, single-base mismatched DNA, three-base mismatched DNA, and non-complementary DNA. $F_{0}$ and $F$ are the fluorescence intensity in the absence and presence of target DNA.

lower than that of other label-free fluorescence methods (shown in Table 2). ${ }^{13,19,22,36}$ In addition, the selectivity of the assay has been investigated by testing the fluorescence response of four kinds of DNA probes including complementary target DNA (H1N1), singlebase mismatched DNA, three-base mismatched DNA and all mismatched (non-complementary) DNA. As indicated in Fig. 6, it can be seen that over a 3-fold fluorescence response was achieved in the presence of the complementary target DNA. However, single-base mismatched DNA, three-base mismatched DNA and all mismatched (non-complementary) DNA did not induce obvious fluorescence responses. The biosensing strategy showed high selectivity and possessed the ability to discriminate basemismatched DNA. Thus, on the basis of guanine proximityinduced fluorescence quenching and proximity-dependent hybridization, a label-free fluorescent platform for the detection of DNA was constructed by designing appropriate DNA sequences.

\section{Conclusions}

In summary, a label-free fluorescent platform has been developed for the sensitive detection of target nucleic acids on the basis of the good fluorescence properties of poly(A)-templated AuNCs coupled with proximity-dependent hybridization and the guanine proximity-induced fluorescence quenching. The

Table 2 The comparison of different fluorescence methods for DNA detection

\begin{tabular}{|c|c|c|c|}
\hline Fluorescence analytical methods & Linear range $(\mathrm{nM})$ & LOD (nM) & References \\
\hline DNA-AuNCs with SYBR Green I & $1-10$ & 0.3 & 13 \\
\hline DNA-AgNC probe & Not given & 10 & 19 \\
\hline $\begin{array}{l}\text { AgNCs with G-quadruplex/hemin } \\
\text { complex }\end{array}$ & $1-100$ & 0.6 & 22 \\
\hline AgNCs with hairpin DNA probes & $10-200$ & 3 & 36 \\
\hline DNA-AuNCs with G-rich DNA proximity & $1-100$ & 0.2 & This work \\
\hline
\end{tabular}


fluorescence assay had some advantages. Firstly, the prepared AuNCs exhibited good fluorescence signals, low toxicity, favorable biocompatibility and good photo-stability. Secondly, G-rich DNA induced fluorescence quenching was low cost. Thirdly, a low background was obtained by using the proximity-dependent hybridization. More importantly, the method could be extended to determine other biomolecules by designing appropriate sequences of DNA-AuNC probes and G-rich DNA probes. Thus, it could provide a label-free sensing platform for biochemical assay and clinical diagnostics.

\section{Conflicts of interest}

There are no conflicts to declare.

\section{Acknowledgements}

This work was financially supported by the National Natural Science Foundation of China (No. U1704153 and 21305119), Plan for Young Excellent Teachers in Universities of Henan Province (No. 2017GGJS100), Plan for Scientific Innovation Talent of Henan Province (No. 2017JR0016), and Nanhu Scholars Program for Young Scholars of XYNU.

\section{References}

1 Y. Tao, M. Q. Li, J. S. Ren and X. G. Qu, Chem. Soc. Rev., 2015, 44, 8636-8663.

2 Y. M. Guo, F. P. Cao, X. L. Lei, L. H. Mang, S. J. Cheng and J. T. Song, Nanoscale, 2016, 8, 4852-4863.

3 Q. F. Yao, T. K. Chen, X. Yuan and J. P. Xie, Acc. Chem. Res., 2018, 51, 1338-1348.

4 L. L. Zhang, J. J. Zhao, H. Zhang, J. H. Jiang and R. Q. Yu, Biosens. Bioelectron., 2013, 44, 6-9.

5 H. B. Wang, H. D. Zhang, Y. Chen and Y. M. Liu, Biosens. Bioelectron., 2015, 74, 581-586.

6 J. T. Petty, J. Zheng, N. V. Hud and R. M. Dickson, J. Am. Chem. Soc., 2004, 126, 5207-5212.

7 X. Tian, X. J. Kong, Z. M. Zhu, T. T. Chen and X. Chu, Talanta, 2015, 131, 116-120.

8 L. Y. Guo, T. Tang, L. S. Hu, M. H. Yang and X. Chen, Sens. Actuators, B, 2017, 241, 773-778.

9 A. Rotaru, S. Dutta, E. Jentzsch, K. Gothelf and A. Mokhir, Angew. Chem., Int. Ed., 2010, 49, 5665-5667.

10 Z. H. Qing, X. X. He, D. G. He, K. M. Wang, F. Z. Xu, T. P. Qing and X. H. Yang, Angew. Chem., Int. Ed., 2013, 52, 9719-9722.

11 N. Goswami, A. Giri, M. S. Bootharaju, P. L. Xavire, T. Pradeep and S. K. Pal, Anal. Chem., 2011, 83, 9676-9680.

12 J. Y. Lian, Q. Liu, Y. Jin and B. X. Li, Chem. Commun., 2017, 53, 12568-12571.

13 Z. Y. Li, Y. T. Wu and W. L. Tseng, ACS Appl. Mater. Interfaces, 2015, 7, 23708-23716.
14 Y. L. Zhang, Y. Huang, J. H. Jiang, G. L. Shen and R. Q. Yu, J. Am. Chem. Soc., 2007, 129, 15448-15449.

15 Y. L. Zhang, Y. Wang, H. B. Wang, J. H. Jiang, G. L. Shen, R. Q. Yu and J. H. Li, Anal. Chem., 2009, 81, 1982-1987.

16 R. Hu, T. Fu, X. B. Zhang, R. M. Kong, L. P. Qiu, Y. R. Liu, X. T. Liang, W. H. Tan, G. L. Shen and R. Q. Yu, Chem. Commun., 2012, 48, 9507-9509.

17 J. Y. Xu, M. Shi, W. T. Chen, Y. Huang, L. N. Fang, L. F. Yao, S. L. Zhao, Z. F. Chen and H. Liang, Chem. Commun., 2018, 54, 2719-2722.

18 Z. M. Ying, H. Y. Xiao, H. Tang, R. Q. Yu and J. H. Jiang, Chem. Commun., 2018, 54, 8877-8880.

19 H. C. Yeh, J. Sharma, J. J. Han, J. S. Martinez and J. H. Werner, Nano Lett., 2010, 10, 3106-3110.

20 Y. L. Zhang, P. F. Pang, J. H. Jiang, G. L. Shen and R. Q. Yu, Electroanalysis, 2009, 21, 1327-1333.

21 M. Torimura, S. Kurata, K. Yamada, T. Yokomaku, Y. Kamagata, T. Kanagawa and R. Kurane, Anal. Sci., 2001, 17, 155-160.

22 L. B. Zhang, J. B. Zhu, S. J. Guo, T. Li, J. Li and E. K. Wang, J. Am. Chem. Soc., 2013, 135, 2403-2406.

23 P. Hu, L. H. Jin, C. Z. Zhu and S. J. Dong, Talanta, 2011, 85, 713-717.

24 L. Wang, J. Q. Tian, H. L. Li, Y. W. Zhang and X. P. Sun, Analyst, 2011, 136, 891-893.

25 D. P. Zhang, R. Fu, Q. Zhao, H. Q. Rong and H. L. Wang, Anal. Chem., 2015, 87, 4903-4909.

26 Y. Saito, E. Mizuno, S. S. Bag, I. Suzukaa and I. Saito, Chem. Commun., 2007, 4492-4494.

27 D. S. Xiang, K. Zhai and L. Z. Wang, Analyst, 2013, 138, 53185324.

28 W. J. Wang, C. L. Chen, M. X. Qian and X. S. Zhao, Sens. Actuators, B, 2008, 129, 211-217.

29 X. Y. Fan, F. B. Lin, Y. Y. Zhang, J. N. Zhao, H. T. Li and S. Z. Yao, New J. Chem., 2012, 36, 2260-2265.

30 C. B. Ma, H. C. Chen, R. Han, H. L. He and W. M. Zeng, Anal. Biochem., 2012, 429, 8-10.

31 X. Su, X. C. Zhu, C. Zhang, X. J. Xiao and M. P. Zhao, Anal. Chem., 2012, 84, 5059-5065.

32 Y. C. Wang, L. Z. Yang, Y. J. Wang, W. Liu, B. X. Li and Y. Jin, Sens. Actuators, B, 2017, 252, 477-482.

33 H. B. Wang, Y. Li, H. Y. Bai and Y. M. Liu, Sens. Actuators, B, 2018, 259, 204-210.

34 H. B. Wang, Y. Li, H. Y. Bai, Z. P. Zhang, Y. H. Li and Y. M. Liu, Food Anal. Method., 2018, 11, 3095-3102.

35 N. R. Markham and M. Zuker, Nucleic Acids Res., 2005, 33, W577-W581.

36 Y. Xiao, Z. J. Wu, K. Y. Wong and Z. H. Liu, Chem. Commun., 2014, 50, 4849-4852. 